\title{
Derrière Les paravents: La vérité selon Jean Genet
}

Carine Bourget

$\boldsymbol{L}$

es Paravents, dernière oeuvre thêâtrale de Jean Genet, a vu s'écouler cinq années entre sa parution en 1961 et sa première représentation par la troupe Jean-Louis Barrault - Madeleine Renaud en 1966, ce qui semble paradoxal lorsque l'on sait l'importance que l'auteur attache à l'aspect visuel. Le thêatre de Genet exploite les différents signes que la représentation permet, et même une analyse purement textuelle ne peut négliger les nombreuses indications scéniques qui parfois contredisent le contenu des dialogues. Cependant, les critiques ont tendance à concentrer leur analyse sur un aspect de la pièce au détriment de l'autre. Ainsi, l'analyse sociologique de Goldman se concentre uniquement sur l'aspect didactique de la thématique de la décolonisation, analyse réfutée par le traitement sémiotique de Chaudhuri parce qu'elle attribue un message clair et cohérent que l'auteur lui-même renie (Chaudhuri 111). Chaudhuri conclut que le theâtre de Genet "does not go anywhere. Instead it creates and maintains a static dynamic, a paradox reflected in its simultaneous affirmation and denial of all signs" (Chaudhuri 127). En effet, certains critiques prennent au pied de la lettre l'un des rares commentaires que l'auteur, beaucoup plus prolifique en ce qui concerne la représentation, a glissé parmi ses conseils à Roger Blin, metteur en scène des Paravents: "Les pièces, habituellement, dit-on, auraient un sens: pas celle-ci. C'est une fête dont les éléments sont disparates, elle n'est la célébration de rien" (cité dans Bickel 127). Cette citation a également influencé Bickel, selon qui Genet construit et dépouille pour mieux montrer "le vide, le néant qui, selon lui, constitue le fondement de l'être" (Bickel 128), ainsi que Dort qui y voit la négation d'un théâtre qui s'autodétruit (Dort 127-8). L'interprétation psychanalitique de Cetta qui affirme que Genet "immerse the audience in evil so that it is forced to face the repressed other in the Self" (Cetta 87) est l'une des plus compréhensives en ce sens qu'elle prend en considération le contenu et la forme. Ces diverses interprétations donnent au vide un sens 
négatif que cet essai se propose d'inverser, en examinant le rapport existant entre le contenu qui entreprend une démystification du pouvoir, et la forme qui remet en question la conception traditionnelle du thêatre. Ce lien constituera le point de départ d'une définition de ce "rien" illusoire dans lequel se rejoignent les "éléments disparates" qui composent Les Paravents.

De par le contexte historique marqué par la chute de l'empire colonial français, Les Paravents a tout d'abord été reçu comme une satire virulente du colonialisme. Les représentations furent interrompues à deux reprises par des émeutes, et le Comité des Relations Publiques des Anciens Combattants d'Indochine et d'Algérie tentèrent de faire retirer la pièce (Genet 1972: 49, note du traducteur). L'anachronisme des costumes -au douzième tableau les Européens portent des costumes de 1840 environ- situe le drame hors du temps et implique que l'attitude des colonisateurs n'a pas évolué depuis 1837. La première référence concernant l'Algérie se trouve dans le septième tableau dans la description vestimentaire du Cadi qui porte un "costume traditionnel algérien"(59). De plus, les noms des colons aux consonnances anglo-saxonnes peuvent évoquer d'autres puissances coloniales. La plupart du temps, les personnages sont présentés comme étant avant tout Arabes et Européens. Le caractère atemporel et générique de la pièce est propre aux agents de la colonisation, qui, quel que soit le pays ou l'époque, se basent toujours sur les mêmes injustices.

La satire des motivations ethnocentristes typiques du colonialisme est évidente dans certaines répliques des colons. Le Gendarme adresse le reproche suivant à Leîla: "On est venu chez vous avec la civilisation et vous continuez à vivre en vagabonds." (88), Blankensee affirme à Sir Harold "c'est des hommes comme nous qui ont fait ce pays" (94). L'auteur dévoile le racisme que cache un mélange ambigu de paternalisme et de préjugés, la dichotomie amour/haine qui est à la base des rapports entre colonie et métropole. Par exemple, le Gendarme prétend fraterniser avec ceux qu'il désigne par le terme péjoratif de "Bicots" (87). Ce double aspect se retrouve dans la révolte des Arabes qui n'est pas un mouvement unifié, il y a un contraste net entre Warda qui désigne la France par le mot "patrie" (25) et Ahmed qui exalte "la haine des étrangers" (27), c'est-àdire des colons. Sir Harold, dans la même réplique, affirme que l'Arabe est intrinsèquement voleur (98) et mentionne le fait que les colons leur ont volé un mot (99): le système de valeurs sur lequel se base le colon est perverti, et il se dénonce dans cette contradiction.

La domination des colons s'exerce par l'illusion, qui, lorsqu'elle sera devenue transparente pour les colonisés, donnera lieu à l'accession au pouvoir de ces derniers. L'instrument du pouvoir de Sir Harold, c'est ce gant qui reste suspendu miraculeusement en l'air , ou plutôt qui ne tient à rien de concret, et qui est décrit par Habib de la façon suivante: "Y a de la paille. Bien bourrée, pour faire comme s'il y avait son poing... [...] Et pour faire que ça paraisse plus dangereux... [...] Et pour faire que ça soit plus vrai..." (42). De même, le prestige de Monsieur Blankensee tient à ses coussinets (95), il reconnaît qu'il faut tout ce truquage pour nous imposer... pour en imposer!" (96). Mais le moyen le plus pernicieux est de se rendre "les maîtres du langage" (97), car celui qui maîtrise le langage -comme Blankensee le prétend par la description de ses roses- pense comprendre la réalité, et peut donc exercer sur celle-ci son propre contrôle. 
Sir Harold est décrit dans les indications scéniques tout au début du quatrième tableau comme étant "très viril selon le langage des colons" (37, je souligne). S'il existe un langage spécifique aux colons, cela implique l'existence d'autres langages, ce moyen d'expression est intrinsèquement subjectif. Pour lui, "un Arabe ne vaut jamais cher" (91), la Vamp cherche les "sauvages"(120). C'est par le pouvoir des mots que Sir Harold a imposé au peuple colonisé l'image qu'il a construite de ce dernier, et qu'il leur impose en lui donnant un caractère inne:

\section{LE FILS}

Oui, qui t'a enseigné le tremblement?

\section{LE CHEF}

La droiture de votre regard et notre nature servile. (128).

L'ironie que l'on devine dans la réplique du chef est signe de l'effondrement du pouvoir des mots et de la révolte qui s'annonce. C'est pour cette raison que le Lieutenant donne l'ordre de tirer d'abord sur les miroirs (164), car leur empire s'effondrera le jour où l'image qu'ils ont construite sera confrontée à la réalité. Saïd et Leïla caricaturent l'image que les colons ont construites des Arabes: la laideur de Leïla n'existe qu'au niveau du discours, son visage sera dissimulé sous une cagoule noire -qui, de façon significative, sera retrouvée alors que Leïla aura disparu et que les Arabes émergent victorieux- et le malheur de Saïd est la honte que lui causent la laideur de sa femme, et par extension l'image qu'il a de luimême. Il existe un décalage, un vide à combler, entre les codes visuel et linguistique qui ne sont pas mimétiques.

La construction illusoire et artificielle sur laquelle la puissance coloniale est basée fait le lien entre le discours colonialiste et l'expression théâtrale. Dans une interview, Jean Genet a déclaré: "It seems as if power could never get along without theatricality. Power protects itself by means of theatricality" (Fichte 182). La dénonciation de l'artifice à la base du pouvoir entraîne un démontage des mécanismes du théâtre, entraînant une prise de conscience aussi bien sur scène chez les colonisés que dans le public.

L'abondance des indications scéniques des Paravents témoigne de l'importance donnée aux objets et à tout ce qui est visuel. D'une part, la multiplicité des objets évoque le théâtre de l'absurde, dans lequel les personnages sont submergés par des objets au statut autonome. Au début du troisième tableau, Leïla s'adresse au pantalon de Saïd comme à un être humain. D'autre part, les "Quelques indications" préliminaires donnent un avant-goût de ce que l'on peut nommer la défaîte du langage chez Genet. L'auteur précise que des dessins accompagneront ses explications de l'agencement de la scène (9), un autre code lui est nécessaire pour exprimer sa vision.

L'auteur utilise le dessin comme code alternatif au langage pour luimême ainsi que pour ses personnages, mais seuls les Arabes dessinent leurs actions sur les paravents: le dessin devient alors symbole de défi. Au douzième tableau, la scène est divisée en deux niveaux de façon à représenter la hiérarchie sociale: sur une plateforme, Monsieur et Madame Bonneuil épinglent fièrement des décorations de guerre aux noms fantaisistes, mais qui sont l'équivalent des 
crimes que les Arabes qui se trouvent en bas dessinent tour à tour sur le paravent. Un autre exemple se situe au dixième tableau, ce sont les flames dessinées par des ouvriers Arabes qui vont "brûler" les orangers. Ces actes sans paroles se trouvent en contre-point du dialogue sur le pouvoir colonial entre Monsieur Blankensee et Sir Harold, ce sont deux discours -colonialisme/indépendantismequi se fondent sur deux codes à la fois semblables et différents, car ils ne sont qu'illusion. Le langage est une construction artificielle sans rapport avec la réalité qu'il est censé dénoter, et à laquelle les Arabes essaient vainement de trouver une alternative: le code qu'ils adoptent a pour base ce qu'ils veulent renverser: l'illusion sémantique.

Les dessins effectués devant les spectateurs sur les paravents sont des mises en abyme de la création artistique, tout comme la séance d'habillage de Warda au deuxième tableau est une mise en abyme du rôle du comédien. Ce procédé, qui consiste à faire assister le spectateur à la mise des costumes est typique de ce qu'Abel nomme métathéâtre, et qui fait paraître réel tout ce qui se trouve sur scène et qu'habituellement nous tenons pour faux (Abel 80). Nous nuancerons cette remarque: le spectateur se trouve en situation de doute et d'incertitude totale par rapport au caractère à la fois réel et illusoire de la mise en abyme qui se déroule sur scène. Tout comme l'association d'éléments opposés le paravent de la maison close est de couleur blanche, couleur associée en général avec la pureté-, l'auteur cherche à provoquer chez son public une mise en question de ce qu'il tient pour certain. L'auteur intervient a plusieurs reprises. S'il se retranche parfois derrière des aphorismes ironiques, tel "Leur [les Arabes] teint sera basané, comme on dit." (10, je souligne), il met en doute l'exactitude d'une comparaison qu'il effectue dans les indications scéniques: "chapeau de feutre à larges bords, comme au Texas, je crois" (168, je souligne). Ce "je" (invisible pour les spectateurs, et que seuls les lecteurs remarqueront) fait le lien entre les deux aspects de la pièce: ce spectacle est la construction d'un dramaturge, qui, tout comme les colonisateurs, utilise l'artifice et l'illusion pour transmettre sa vision du monde.

L'artifice et le réel sont juxtaposés, tout d'abord les "maquillages excessifs contrast[e]nt avec le réalisme des costumes" (10); ceci dans le but de souligner le côté doublement artificiel du thêâtre, en remettant en question ce que l'on tient pour réel: "Auprès du paravent, il devra toujours y avoir au moins un objet réel [...], destiné à confronter sa propre réalité avec les objets dessinés" (10). Ainsi, le moindre signe se manifestant pendant une représentation théâtrale est investi d'un certain sens, l'auteur nous dit lui-même qu'il a déjà interprété ce signe (Genet 1972: 25).

Les rapports qui lient les groupes apparemment distincts Arabescolonisés/Européens-colons, l'artiste/le public, l'homme/la femme, sont basés sur un pouvoir illusoire, et il en est de même pour les oppositions d'abstractions vie/mort, amour-sexe/guerre. Cependant, cette conception binaire du monde se révèle obsolète. Dans le treizième tableau, le Lieutenant est dans l'impossibilité de déduire d'après le visage de Pierre si ce dernier est Arabe ou Français (153154). Le phénomène d'acculturation est notable au niveau des costumes, ceux des Arabes forment un patchwork aux couleurs vives, cependant peu d'entre eux portent des costumes traditionnels. Dans le dixième tableau, l'auteur insiste à plusieurs reprises sur les costumes des ouvriers arabes qui dessinent le feu sur les orangers: ils "seront vêtus de costumes coupés à l'européenne, mais de couleurs vives et disparates" (90). L'influence étrangère ne se manifeste pas qu'au niveau vestimentaire. Les opprimés reproduisent les structures de leurs oppresseurs 
après s'être révoltés contre elles, comme le montre cet échange:

LE ler COMBATTANT (montrant les Européens)

Et ceux-là, qu'est-ce qu'ils vont penser de toi... et de nous?

\section{LA MERE (éclatant de rire)}

Mais puisque c'est ceux-là que vous voulez démolir! Tu crois que leurs dorures, c'est pas du bidon?...(258).

Le combattant se rend compte que "d'ici peu, [ils] seron[t] tous cartésiens" (255), c'est-à-dire que la façon de penser européenne s'est subrepticement infiltrée dans leur esprit. Ce n'est pas un hasard si le prénom de Warda signifie "une rose" en arabe et si les fleurs que Monsieur Blankensee cultive sont des roses. Warda est inclassable: le pouvoir érotique qu'elle exerce sur les hommes est basé lui aussi sur l'artifice, elle duplique le pouvoir auquel son peuple est soumis.

Ce croisement entre groupes n'est pas omniprésent, il est parfois remplacé par une distance infranchissable. Le langage et les objets se rejoignent en ce sens qu'ils construisent le vide. La valise que porte la mère au premier tableau, "pleine de cadeaux" (15), "tombe par terre en s'ouvrant, et perd tout son contenu: elle était vide" (20). Les deux systèmes linguistique et visuel sont en contradiction, de plus le vide devient un objet. Les contradictions existent également au niveau interne, et elles apparaissent à deux reprises au niveau visuel: "Tous les morts baissent la tête (alors que la Mère est au-dessus d'eux) pour la regarder"(188, indications scéniques). Les vêtements de Warda sont analogues à la valise. Elle qui a pratiqué la prostitution comme un art(ifice), elle n'existe que par ses atours et son style, c'est par ses artifices qu'elle fascine et domine les hommes. Warda, tout comme les colons une fois leur pouvoir perdu, se retrouve en loques; les Arabes, qui ne se laissent plus leurrer par les apparences, l'obligent à se défaire de ses atours. Warda, qui tirait sa fierté de ce style -vêtements et curage de dents- qu'elle a mis vingt ans à perfectionner, est maintenant obligée de se mettre à nu comme les prostituées françaises pour ses clients. Paradoxalement, les Arabes s'occidentalisent dans leurs moeurs sexuelles alors qu'ils viennent de se dégager de la tutelle française. Cette occidentalisation se traduit par un renversement: Warda dominait, elle est maintenant dominée. L'essence du pouvoir est donc dans l'illusion, et s'il change de main, il semble que l'opposition binaire dominants/dominés soit immuable, ${ }^{1}$ puisque les Arabes reproduisent les mêmes structures que leurs oppresseurs.

Genet se sert de l'illusion qui est à la base même de la représentation pour dénoncer l'ordre établi. En dévoilant les mécanismes du théâtre, Genet refuse de donner à sa pièce l'apparence du vrai propre au théâtre traditionnel. Le dramaturge cultive le côté magique de l'illusion thêâtrale dans l'invraisemblance de certaines indications scéniques impossibles à mettre en pratique (par exemple: "Sur le paravent apparaît un croissant de lune" 49).

La frontière entre la réalité et l'illusion est symbolisée par les paravents qui donnent son titre à ce spectacle déconcertant. En tant qu'éléments du décor, ils sont cet espace réel sur lequel s'inscrit l'illusion, une frontière artificielle entre la vie et la mort, un entre-deux ineffable car il est inconcevable. A la suite des personnages, Genet invite son public à briser les paravents. Tout comme l'espace scénique est dépouillé de tout artifice -les acteurs emportent les derniers 
objets-, la pièce laisse une impression d'absence de signification: ce vide est la vérité dépouillée de tout artifice, absence de vérité ou d'une vérité. Selon Jean Genet,

it is only that kind of truths -the truths that cannot be proven and that, in fact, are false, those that one cannot, without seeming absurd, carry to their ultimate conclusions without arriving at the negation both of the truths themselves and of oneself -these are the truths that ought to be exalted by any work of art (Genet 1972: 77).

La pièce Les Paravents est à l'image de son décor: l'auteur précise qu'il veut une scène close, délimitée, dans un théâtre en plein air: à la fois clôture et ouverture. Genet laisse libre cours à l'imagination de son public qu'il n'emprisonne pas dans sa propre vision. 


\section{Notes}

'Les autres pièces de Genet (Les Bonnes, Le Balcon, Les Nègres) mettent en relief la même structure.

\section{Ouvrages cités}

Abel, Lionel. Metatheatre. A New View of Dramatic Form. New York: Hill and Wang, 1963.

Bickel, Gisèle. Jean Genet. Criminalité et transcendance. Stanford French and Italian Studies 55. Saratoga, Calif.: Anma Libri, 1987.

Cetta, Lewis. Profane Play, Ritual, and Jean Genet. Alabama: The University of Alabama Press, 1974.

Chaudhuri, Una. No Man's Stage. A Semiotic Study of Jean Genet's Major Plays. Ann Arbor, Michigan: UMI Research Press, 1986.

Dort, Bernard. "Genet: The Struggle with Theater." Genet. A Collection of Critical Essays. Ed. Peter Brooks and Joseph Halpern. Englewood Cliffs, N.J.: Prentice-Hall, 1979.

Genet, Jean. Les Paravents. Paris: L'Arbalète, 1961.

- Reflections on the Theatre and Other Writings. Trad. Richard Seaver. London: Faber \& Faber, 1972.

Goldman, Lucien. "The theater of Genet: A Sociological Study." Genet. A Collection of Critical Essays. Peter Brooks and Joseph Halpern, Eds. Englewood Cliffs, N.J.: Prentice-Hall, 1979.

Fichte, Hubert. "I Allow Myself to Revolt: Interview with Jean Genet." Genet. A Collection of Critical Essays. Peter Brooks and Joseph Halpern, Eds. Englewood Cliffs, N.J.: Prentice-Hall, 1979. 
